Pacific Journal of Mathematic 


\title{
LIPSCHITZ FUNCTIONS OF CONTINUOUS FUNCTIONS
}

\author{
Imanuel MarX and George Piranian
}

1. Introduction. The present paper was suggested by a note of W. S. Loud [2] in which the following theorem on functions of a real variable is proved.

THEOREM 1. If $\alpha$ is a constant $(0<\alpha<1)$, there exist a continuous function $f(t)$ and a pair of positive constants $K_{1}$ and $K_{2}$ such that

$$
|f(t+h)-f(t)|<K_{1}|h|^{a}
$$

for all $t$ and all $h$, and such that

$$
\limsup _{h \rightarrow 0} \frac{|f(t+h)-f(t)|}{|h|^{\alpha}}>K_{2}
$$

for all $t$.

It is natural to examine the possibility of a variable exponent $\alpha(t)$ and to consider various definitions that associate with every continuous function $f(t)$ a "Lipschitz function" $a(t ; f)$. For a reasonable choice of the definition, Loud's result implies that every constant $\alpha(0<\alpha<1)$ is the Lipschitz function of some continuous function. The following sections offer two different definitions of Lipschitz functions, and deal with the problem of characterizing the functions that are Lipschitz functions of continuous functions.

2. The point Lipschitz function of a function. Let $f(t)$ be a continuous, real-valued function of $t$. Consider the quantity

$$
Q\left(\alpha, t_{0} ; f\right)=\limsup _{h \rightarrow 0} \frac{\left|f\left(t_{0}+h\right)-f\left(t_{0}\right)\right|}{|h|^{\alpha}} .
$$

If $Q\left(\alpha, t_{0} ; f\right)$ is finite for $\alpha=\alpha^{\prime}$, it is zero for all $\alpha$ less than $\alpha^{\prime}$; if $Q$ is greater than zero for $\alpha=\alpha^{\prime}$, it has the value $+\propto$ for all $\alpha$ greater than $\alpha^{\prime}$. Let $\alpha\left(t_{0} ; f\right)$ denote the least upper bound of all $\alpha$ for which $Q\left(\alpha, t_{0} ; f\right)$ is

Received November 26, 1951.

Pacific J. Math. 3 (1953), 447-459 
finite. Then $\alpha(t ; f)$ shall be called the point Lipschitz function of $f(t)$. A simple computation shows that

$$
\alpha(t ; f)=\liminf _{h \rightarrow 0} \frac{\log |f(t+h)-f(t)|}{\log |h|},
$$

where the fraction on the right is to be interpreted as having the value $+\infty$ whenever $f(t+h)=f(t)$.

THEOREM 2. If $f(t)$ is continuous $(-\infty<t<\infty)$, then $\alpha(t ; f)$ is the inferior limit of a sequence of continuous functions of $t$; if $\alpha(t ; f)>1$ throughout sonie open interval of the t-axis, then $\alpha(t ; f)=\infty$ throughout the interval.

Let $L(t, h ; f)$ denote the fraction in the right member of $(1)$, let $\epsilon$ denote a constant $(0<\epsilon<1 / 2)$, and let

$$
\alpha_{\epsilon}(t ; f)=\min \left[1 / \epsilon, \min _{\epsilon \leq|h| \leq 2 \epsilon} L(t, h ; f)\right] .
$$

Then $\alpha_{\epsilon}(t ; f)$ is a continuous function of $t$, because of the restriction on $h$ and the truncation of $L(t, h ; f)$ imposed in the right member of (2). If $\epsilon$ is assigned the successive values $1 / 4,1 / 8,1 / 16, \cdots$, the first part of Theorem 2 follows from the formula (2).

For the second part of the theorem, consider an open interval $I$ on the $t$-axis throughout which $\alpha(t ; f)>1$. Because $f^{\prime}(t)=0$ throughout $I, f(t)$ is constant in $I$, and the proof of the theorem is complete.

THEOREM 3. Let $\left\{\alpha_{n}(t)\right\}$ be a sequence of continuous functions ( $0 \leq$ $\left.\alpha_{n}(t) \leq 1\right)$, and let

$$
\alpha(t)=\lim _{n \rightarrow \infty} \inf \alpha_{n}(t) .
$$

Then there exists a continuous function $f(t)$ such that $\alpha(t ; f)=\alpha(t)$.

The theorem will be proved by a construction analogous to that used by Loud in his proof of Theorem 1 . Let $g(t, s)$ be the continuous function which takes the value 0 when $t$ is an even multiple of $s$, takes the value $l$ when $t$ is an odd multiple of $s$, and is linear between consecutive multiples of $s$. Let $\alpha$ be a constant between 0 and 1 , and let $A>[2(1-\alpha)]^{-1}$ be an integer. Loud proved that the series 


$$
\sum_{n=1}^{\infty} 2^{-2 A a n} g\left(t, 2^{-2 A n}\right),
$$

converges to a function $f(t)$ which has the properties promised in Theorem 1 . Roughly, the principal intuitive idea behind Loud's proof is that for every pair of values $t$ and $h$ at most one or two terms of the series (3) make a significant contribution to the difference $f(t+h)-f(t)$; the contribution is always small enough so that the first inequality in Theorem 1 is satisfied; and for every $t$ there exist arbitrarily small values of $h$ for which the contribution is so large that the second inequality in Theorem 1 is satisfied. The following three sections will be devoted to elaborations of Loud's method that lead to a proof of Theorem 3. We first summarize our construction of a continuous function $f(t)$ whose Lipschitz function $\alpha(t)$ is the inferior limit of a given sequence of continuous functions $a_{n}(t)$. The construction is then described in full detail in the following two sections.

The function $f(t)$ will be written as an infinite series

$$
f(t)=\sum_{m=1}^{\infty} G_{m}(t)
$$

with $G_{m}$ depending on the function $\alpha_{m}(t)$ alone. For every $m$, we divide the $t$-axis into intervals $I$ over each of which the function $\alpha_{m}(t)$ lies within fixed bounds to be specified. The function $G_{m}(t)$ is defined separately in each interval. Over a fixed interval $l$, the graph $\Gamma_{m}$ of the function $G_{m}(t)$ consists of rows of saw-teeth completely filling $l$. There is a row of relatively high and wide teeth in the central portion of the interval, flanked by two rows of somewhat lower and much narrower teeth, which are in turn flanked by two rows of still lower and narrower teeth, and so on. All the teeth of the central row have equal height and equal width, all the teeth of the two flanking rows have equal height and equal width, and so on. Toward the end-points of the interval $l$, the heights and the widths of the teeth of $\Gamma_{m}$ approach zero. The function $G_{m}(t)$ is continuous for all $t$, is differentiable except at a countable number of points, and is not constant in any interval. The heights and the widths of the saw-teeth are so chosen that

$$
\alpha(t ; f)=\alpha(t)
$$

for the function

$$
f(t)=\sum_{m=1}^{\infty} G_{m}(t) .
$$


Details of the proof follow.

3. Classes of intervals. For each $m$, we denote by $I_{m}$ a class of intervals to be constructed, with $I_{m}$ depending on $I_{1}, I_{2}, \ldots, I_{m-1}$ as well as on the functional values of $\alpha_{m}(t)$. The class $I_{1}$ consists of finitely many or infinitely many disjoint, open intervals that meet the following three requirements: each point of the $t$-axis lies in the closure of one of the intervals; no point of the $t$-axis is a limit point of end-points of intervals of the class $I_{1}$; and throughout each of the intervals one of the conditions

$$
\begin{aligned}
0 & \leq \alpha_{1}(t) \leq 3 / 4, \\
1 / 4 & \leq \alpha_{1}(t) \leq 1
\end{aligned}
$$

is satisfied.

When the classes $I_{1}, I_{2}, \ldots, I_{m-1}$ of intervals have been defined, we choose the intervals of the class $I_{m}$ subject to the following four requirements: each point of the $t$-axis lies in the closure of one of the intervals of the class; no point of the $t$-axis is a limit point of end-points of intervals of the class; throughout each interval of the class, one of the conditions

$$
\begin{aligned}
0 & \leq \alpha_{m}(t) \leq 3 / 2^{m+1}, \\
1 / 2^{m+1} & \leq \alpha_{m}(t) \leq 5 / 2^{m+1}, \\
3 / 2^{m+1} & \leq \alpha_{m}(t) \leq 7 / 2^{m+1}, \\
\ldots \ldots \ldots & \ldots \ldots \cdots \\
1-3 / 2^{m+1} & \leq \alpha_{m}(t) \leq 1
\end{aligned}
$$

is satisfied; and no end-point of an interval of the class $I_{m}$ is an end-point of an interval of a previously defined class.

4. The saw-tooth functions. The function $f(t)$ to be constructed will be of the form

$$
f(t)=\sum_{m=1}^{\infty} G_{m}(t)=\sum_{n=1}^{\infty} g_{n}(t)
$$

where 


$$
\begin{aligned}
& G_{1}(t)=g_{1}+g_{3}+g_{6}+g_{10}+\cdots, \\
& G_{2}(t)=g_{2}+g_{5}+g_{9}+\cdots, \\
& G_{3}(t)=g_{4}+g_{8}+\cdots, \\
& G_{4}(t)=g_{7}+\cdots,
\end{aligned}
$$

The indices $n=1,3,6,10, \cdots$, are said to belong to $m=1$, the indices $n=2$, $5,9, \cdots$, are said to belong to $m=2$, and so on. The graphs of the functions $G_{m}(t)$ and $g_{n}(t)$ will be denoted by $\Gamma_{m}$ and $\gamma_{n}$, respectively.

We summarize the construction of the functions $g_{n}(t)$. Corresponding to the function $G_{m}$ we have selected a class $I_{m}$ of intervals, and throughout each interval $I$ of the class $I_{m}$ the function $\alpha_{m}(t)$ satisfies one of $2^{m}$ inequalities specified in $\$ 3$. We choose a class of exponents $c$ in accordance with these inequalities, so that $c$ is fixed throughout each interval $I$, and a decreasing sequence $\left\{s_{n}\right\}$ of positive numbers, where $s_{n}$ depends only on $s_{1}, s_{2}, \ldots, s_{n-1}$ and on the value of $m$ to which the index $n$ belongs. The function $g_{n}(t)$ is continuous, with a graph $\gamma_{n}$ consisting alternately of rows of equally high and equally wide saw-teeth and of segments of the $t$-axis. There are at most two subintervals of each interval $I$ of the class $I_{m}$ where $g_{n}(t)$ differs from zero, and in these subintervals it has the form

$$
g_{n}(t)=s_{n}^{c} g\left(t, s_{n}\right) \text {, }
$$

with the exponent $c$ corresponding to the interval $l$. (The function $g(t, s)$ was defined in $\$ 2$, immediately after the statement of Theorem 3.) The terms $g_{n}(t)$ of the series for $G_{m}(t)$ are so chosen that every point interior to an interval of the class $I_{m}$ lies on the base of a tooth of the graph $\Gamma_{m}$ of $G_{m}(t)$. It remains to describe the choice of the exponents $c$ in $(6)$, to select the sequence $\left\{s_{n}\right\}$, and to determine the position of the teeth in $\gamma_{n}$.

Let $g_{n}(t)$ be a term of $G_{m}(t)$, and let $I$ be an interval of the class $I_{m}$. The graph $\gamma_{n}$ of $g_{n}(t)$ shall contain rows of equal saw-teeth over at most two subintervals of $l$, and the exponent $c$ in (6) determining the height of the teeth shall have the value $1 / 2^{m+1}, 3 / 2^{m+1}, \ldots$, or $1-1 / 2^{m+1}$, according as the function $\alpha_{m}(t)$ satisfies in $l$ the first, second, $\cdots$, or last of the conditions (4). It follows that the height $s_{n}^{c}$ of a tooth in $\gamma_{n}$ has one of finitely many values and depends only on the range of values taken by $\alpha_{m}(t)$ in the interval $I$ where 
the tooth appears, while the width $2 s_{n}$ is the same for all teeth appearing anywhere in $\gamma_{n}$. It also follows that the derivative $g_{n}^{\prime}(t)$ exists (except at denumerably many points), and takes only finitely many values.

The number $s_{1}$ can be chosen arbitrarily, subject to the condition $0<s_{1}<1$. Once the numbers $s_{1}, s_{2}, \ldots, s_{n-1}$ are determined, $s_{n}$ is chosen subject to the following three conditions:

1) We require that $s_{n-1}$ be an even multiple of $s_{n}$.

2) We require that the inequality

$$
s_{n}^{2^{-m-1}}<\frac{s_{n-1}}{10}
$$

be satisfied, where $m$ refers to the function $G_{m}(t)$ of which $g_{n}(t)$ is a term. If this requirement is met, the height of each tooth in $\gamma_{n}$ is no greater than $1 / 20$ the base width of any tooth in $\gamma_{r}(r=1,2, \cdots, n-1)$, and every tooth in $\gamma_{n-1}$ is more than 10 times as high as every tooth in $\gamma_{n}$. It follows that the series $\sum_{n=1}^{\infty}\left|g_{n}(t)\right|$ converges uniformly on the interval $(-\infty, \infty)$.

3) We require finally that the slopes of the sides of the lowest teeth in $\gamma_{n}$ be in absolute value greater than 10 times the sum of the greatest slopes that can possibly occur in $\gamma_{r}(r=1,2, \ldots, n-1)$. Because the side of a tooth of height $s^{c}$ and width $2 s$ has a slope numerically equal to $1 / s^{1-c}$, this requirement is met provided $s_{n}$ is chosen small enough.

We turn now to the disposition of the teeth in $\gamma_{n}$. Let $l$ be any interval of the class $I_{1}$. Then $\gamma_{1}$ shall have as many teeth in $I$ as possible, subject to the restriction that the distance from either end of $I$ to any tooth of the graph shall be greater than twice the height of the tooth.

Again, if $I$ is an interval of the class $I_{1}$, and if $\gamma_{1}$ has no teeth in $I$, then $\gamma_{3}$ shall have as many teeth in $I$ as possible, subject again to the restriction mentioned above. If $\gamma_{1}$ has beeth in $I$, then $\gamma_{3}$ shall have, in $I$, two rows of teeth flanking the row of teeth of $\gamma_{1}$; again, the distance from either end of $I$ to any tooth in $\gamma_{3}$ shall be greater than twice the height of the tooth.

Next, if $I$ is an interval of the class $I_{1}$, then $\gamma_{6}$ shall have teeth in the middle portion of $I$ provided that $\gamma_{3}$ has no teeth in $I$ and $I$ is sufficiently long. If $\gamma_{3}$ has teeth in $I$, then $\gamma_{6}$ shall have two rows of teeth: each of these rows shall be adjacent to a previously constructed row, and shall extend as near as possible to the nearer end of $l$, subject to the condition that the remaining distance be greater than twice the height of the teeth. 
The construction of $\gamma_{n}(n=10,15,21, \ldots)$ proceeds according to the pattern that has been described. The construction of $\gamma_{n}(n=2,5,9, \ldots)$ is similar to the previous construction, with these modifications: the construction is carried out with reference to intervals of the class $I_{2}$, and with reference to the values of the function $\alpha_{2}(t)$; and the distance from each tooth to the end of the interval of the class $I_{2}$ in which it stands is required to be $2^{2}$ times the beight of the tooth $\left(2^{m}\right.$ times the height of the tooth in the construction of teeth belonging to the graph $\Gamma_{m}$ ). The construction of $\Gamma_{m}$ is entirely independent of the construction of $\Gamma_{k}(k \neq m)$. Further details are superfluous, and we must only prove that the function defined by (6) has the required properties.

5. Arithmetical estimates. First we show that if $t_{0}$ is a fixed point, $\alpha_{0}=$ $\alpha\left(t_{0}\right)$, and $\epsilon>0$, then

$$
\limsup _{h \rightarrow 0} \frac{\left|f\left(t_{0}+h\right)-f\left(t_{0}\right)\right|}{|h|^{a_{0}+\epsilon}}=\infty \text {. }
$$

In other words, the point Lipschitz function of $f(t)$ has at $t_{0}$ a value not greater than $C_{0}$.

Let $m$ be any integer such that $t_{0}$ is interior to an interval of the class $I_{m}$ (at most one positive integer $m$ fails to satisfy this requirement); then there exists an integer $n$ such that $g_{n}(t)$ is a term in the series defining $G_{m}(t)$ and such that $t_{0}$ lies on the base of a tooth of the graph $\gamma_{n}$ of $g_{n}(t)$. Therefore $g_{n}(t)$ is linear in a sufficiently small interval with $t_{0}$ as end-point; that is, there exists a number $h$, with

$$
\frac{1}{2} s_{n} \leq|h|<s_{n},
$$

such that the function $g_{n}(t)$ is linear in the interval joining the points $t=t_{0}$ and $t=t_{0}+h$. For this number $h$ we have further

$$
\left|G_{m}\left(t_{0}+h\right)-G_{m}\left(t_{0}\right)\right|=\left|g_{n}\left(t_{0}+h\right)-g_{n}\left(t_{0}\right)\right|=|h| s_{n}^{c-1}
$$

where

$$
c<\alpha_{0}+2^{-m}+2^{-m-1}
$$

For all teeth that cover the segment joining $t_{0}$ and $t_{0}+h$ and belong to graphs $\gamma_{r}$.with $r<n$, the requirement 3$)$ on $\left\{s_{n}\right\}$ implies that 


$$
s_{n}^{c-1}>10 \sum_{r<n} \max \left|g_{r}^{\prime}(t)\right|
$$

and therefore that

$$
\sum_{r<n}\left|g_{r}\left(t_{0}+h\right)-g_{r}\left(t_{0}\right)\right|<|h| s_{n}^{c-1} / 10
$$

For the functions $g_{r}$ with $r>n$, the requirement 2 ) on $\left\{s_{n}\right\}$ gives

$$
\sum_{r>n}\left|g_{r}\left(t_{0}+h\right)-g_{r}\left(t_{0}\right)\right|<s_{n}(1 / 10+1 / 100+\cdots)<s_{n} / 5
$$

From (8) and from the estimates obtained thus far it follows that

$$
\begin{aligned}
\frac{\left|f\left(t_{0}+h\right)-f\left(t_{0}\right)\right|}{|h|^{a_{0}+\epsilon}} & >\frac{|h| s_{n}^{c-1}-|h| s_{n}^{c-1} / 10-s_{n} / 5}{|h|^{a_{0}+\epsilon}} \\
& >s_{n}^{c}(9 / 20-1 / 5) s_{n}^{\alpha_{0}+\epsilon}=\frac{1}{4} s_{n}^{c-a_{0}-\epsilon} .
\end{aligned}
$$

By (9), the exponent in the last member is less than $-\epsilon / 2$ if $m$ is large enough, and therefore the relation ( 7 ) is established.

Secondly we must prove that, for every $\epsilon>0$,

$$
\limsup _{h \rightarrow 0} \frac{\left|f\left(t_{0}+h\right)-f\left(t_{0}\right)\right|}{|h|^{a_{0}-\epsilon}}=0 .
$$

We choose an integer $m_{0}$ such that $2^{-m}+2^{-m-1}<\epsilon$ for all $m>m_{0}$, and a positive quantity $h_{0}$ such that the interval

$$
t_{0}-h_{0} \leq t \leq t_{0}+h_{0}
$$

contains no end-point of an interval of any of the classes $I_{1}, I_{2}, \ldots$, or $I_{m_{0}}$, except possibly the point $t_{0}$ itself. Without restricting the generality of the proof, we suppose that $|h|<h_{0}$.

To establish (10), we make separate estimates of the variation of $G_{m}(t)$ 
for the following three cases: the point $t_{0}$ is an end-point of an interval of the class $I_{m} ; t_{0}$ is not an end-point, and $m \leq m_{0}$; or $t_{0}$ is not an end-point, and $m>m_{0}$.

If $t_{0}$ is an end-point of an interval of the class $I_{m}$, then the disposition of the saw-teeth ensures that

$$
\left|G_{m}\left(t_{0}+h\right)-G_{m}\left(t_{0}\right)\right|<|h| / 2^{m}
$$

For each $m \leq m_{0}$ for which $t_{0}$ is not an end-point of an interval of the class $I_{m}$, the quantity $G_{m}\left(t_{0}+h\right)-G_{m}\left(t_{0}\right)$ can be written as the sum of finitely many terms of the form $g_{n}\left(t_{0}+h\right)-g_{n}\left(t_{0}\right)$; the set of indices $n$ that occur in this sum depends only on $h_{0}$, not on $h$. Since the corresponding derivatives $g_{n}^{\prime}(t)$ are bounded, it follows that, for every $\alpha<1$,

$$
\lim _{h \rightarrow 0} \sum_{m \leq m_{0}} \frac{\left|G_{m}\left(t_{0}+h\right)-G_{m}\left(t_{0}\right)\right|}{|h|^{\alpha}}=0 .
$$

For $m>m_{0}$ and $|h|<h_{0}$, two possibilities arise, for each index $m$ : either $t_{0}$ and $t_{0}+h$ both lie in the same interval of the class $I_{m}$, or they do not lie in the same interval. In the latter case, the inequality (11) holds, and it is therefore sufficient to discuss the former case.

Let $k$ be the least integer such that $s_{k} \leq|h|$ and such that the term $g_{k}(t)$ occurs in the series defining a function $G_{m}(t)$ with $m>m_{0}$. The choice of the exponents in the definition (6) of $g_{n}(t)$, and the requirement 2 ) on the sequence $\left\{s_{n}\right\}$, imply the inequality

$$
\sum_{r \geq k}\left|g_{r}\left(t_{0}+h\right)-g_{r}\left(t_{0}\right)\right|<2 s_{k}^{c} \leq 2|h|^{c},
$$

and the quantity $c$ is greater than $\alpha_{0}-\epsilon$ because of our choice of $m_{0}$. Finally we estimate the contribution from those terms $g_{r}(t)$, occurring in the series that define functions $G_{m}(t)$ with $m>m_{0}$, for which $s_{r}>|h|$ while $t_{0}$ and $t_{0}+h$ lie in the same interval of the class $I_{m}$. Let $p$ be the greatest value of the index $r$ for such terms. We find

$$
\left|g_{p}\left(t_{0}+h\right)-g_{p}\left(t_{0}\right)\right|<|h| s_{p}^{c-1}<|h|^{c}\left|s_{p} / h\right|^{c-1}<|h|^{c},
$$

and the sum of the remaining terms of the same category is less than half of the last member. The inequality (10) is established, and the proof of Theorem 3 is 
complete.

We observe that Theorem 3 no longer holds if the restriction $0 \leq \alpha_{n}(t) \leq 1$ is removed. For if $f(t)$ is a continuous function, the set of points where $\alpha(t ; f) \leq 1$ cannot have isolated points. The complete characterization of the functions that are point Lipschitz functions of continuous functions appears to be difficult.

6. The local Lipschitz function of a function. Let $f(t)$ be a continuous, real-valued function of $t$, and let $h$ be a variable taking positive values. Denote by $B\left(t_{0}, h ; f\right)$ the least upper bound of all numbers $\beta$ for which the quantity

$$
\frac{\left|f\left(t^{\prime \prime}\right)-f\left(t^{\prime}\right)\right|}{\left(t^{\prime \prime}-t^{\prime}\right)^{\beta}}
$$

remains bounded as long as the variables $t^{\prime}$ and $t^{\prime \prime}$ satisfy the restriction $t_{0}-h \leq t^{\prime}<t^{\prime \prime} \leq t_{0}+h$. For each value $t_{0}, B\left(t_{0}, h ; f\right)$ is a nonincreasing function of $h$. The quantity

$$
\beta(t)=\beta(t ; f)=\lim _{h \rightarrow 0^{+}} B(t, h ; f)
$$

shall be called the local Lipschitz function of $f(t)$.

It follows at once from the definition that

$$
\beta(t ; f) \leq \alpha(t ; f)
$$

for every continuous function $f(t)$. That equality does not always occur is seen from the following example. Consider the function

$$
f(t)=t \sin 1 / t(t \neq 0), f(0)=0
$$

In every closed interval that does not contain $t=0, f(t)$ has a bounded derivative, so that $\alpha(t ; f) \geq 1$ for $t \neq 0$. Since $\alpha(0 ; f)=1$, it follows that the point Lipschitz function of $f(t)$ is everywhere equal to 1 [except at the zeros of $f^{\prime}(t)$, where $\left.\alpha(t ; f)=2\right]$. On the other hand, the local Lipschitz function $\beta(t ; f)$ has the value 1 everywhere except at $t=0$, and $\beta(0 ; f)=1 / 2$ (for details, see [1]). It follows that equations (1) and (12) do not define equivalent Lipschitz functions.

7. Characterization theorems. The following two theorems provide a characterization of bounded local Lipschitz functions of continuous functions. 
THEOREM 4. If $f(t)$ is continuous $(-\infty<t<\infty)$, then

i) $\beta(t ; f)$ is lower semi-continuous;

ii ) for each point $t$, either $0 \leq \beta(t ; f) \leq 1$, or $\beta(t ; f)=\infty$;

iii) the set of points $t$ where $\beta(t ; f) \neq \propto$ is a perfect set.

Suppose that $\beta(t)=\beta(t ; f)$ is the local Lipschitz function of a continuous function $f(t)$. If $\beta\left(t_{0}\right)>1$, the point $t_{0}$ is interior to an interval in which $f(t)$ satisfies a Lipschitz condition with an exponent greater than 1 . Then $f(t)$ is constant in an interval about $t_{0}$, so that $\beta\left(t_{0} ; f\right)=\infty$. Part ii) of the theorem is proved.

The set of points where $0 \leq \beta(t ; f) \leq 1$ cannot have isolated points, and the set of points where $\beta(t ; f)=\infty$ is open. Therefore part iii) of the theorem is proved, as well as part $\mathrm{i}$ ) for those points $t$ where $\beta$ is infinite. Finally, if $\beta\left(t_{0}\right) \leq 1$, for every $\epsilon>0$ there exists an interval $\left|t-t_{0}\right|<\delta$ in which

$$
\beta(t)>\beta\left(t_{0}\right)-\epsilon \text {. }
$$

It follows that

$$
\beta\left(t_{0}\right) \leq \lim _{t \rightarrow t_{0}} \inf \beta(t)
$$

for all $t_{0}$, and the proof of the theorem is complete.

THEOREM 5. Let $\beta(t)(0 \leq \beta(t) \leq 1)$ be a lower semi-continuous function. Then there exists a strictly increasing continuous function $F(t)$ such that

$$
\beta(t ; F)=\beta(t)
$$

Let $\left\{I_{r}\right\}$ denote a sequence of closed intervals on the $t$-axis with the following property: for each point $t_{0}$ and for every $\epsilon>0$, there exists an interval $I_{r}$ of length less than $\epsilon$, covering $t_{0}$. In each interval $I_{r}$ we select a point $t_{r}$ at which $\beta(t)$ assumes its minimum value in the interval. The function $F(t)$ will be chosen as an infinite series

$$
F(t)=\sum_{r=1}^{\infty} f_{r}(t) .
$$

If the point $t_{r}$ coincides with one of the points $t_{1}, t_{2}, \cdots, t_{r-1}$, we choose 
$f_{r}(t) \equiv 0$; otherwise we choose $f_{r}(t)$ as a strictly increasing function of $t$ whose local Lipschitz function has the value $\beta\left(t_{r}\right)$ for $t=t_{r}$ and the value 1 everywhere else.

The term $f_{r}(t)$ is constructed from the function

$$
f(t ; \gamma)=\frac{|t|^{\gamma} \operatorname{sgn} t}{1+|t|^{\gamma}}
$$

This function is strictly increasing; for

$$
f^{\prime}(t ; \gamma)=\frac{|t|^{\gamma-1}}{\left(1+|t|^{\gamma}\right)^{2}},
$$

when $t \neq 0$, and the function is continuous at $t=0$. Furthermore, the local Lipschitz function of $f(t ; \gamma)$ has the value $\gamma$ at $t=0$, since

$$
\left|f\left(t^{\prime \prime} ; \gamma\right)-f\left(t^{\prime} ; \gamma\right)\right| \leq 2\left|t^{\prime \prime}-t^{\prime}\right|^{\gamma}
$$

for all distinct $t^{\prime}$ and $t^{\prime \prime}$; and it has the value 1 everywhere else, since $f^{\prime}(t ; \gamma)$ is continuous for $|t|>0$. In order to adapt $f(t ; \gamma)$ to our needs we require a sequence $\left\{\rho_{r}\right\}$ of positive numbers, chosen as follows. We set $\rho_{1}=1$. If $t_{r}$ coincides with $t_{1}, t_{2}, \cdots$, or $t_{r-1}$, the function $f_{r}(t)$ is identically zero, and no number $\rho_{r}$ is needed. If all the quantities $\left|t_{r}-t_{1}\right|,\left|t_{r}-t_{2}\right|, \ldots,\left|t_{r}-t_{r-1}\right|$ $(r>1)$ exceed 1 , we set $\rho_{r}=1$; otherwise we set $\rho_{r}$ equal to the least of these quantities.

The nonzero terms $f_{r}(t)$ of the function $F(t)$ are given by the formula

$$
f_{r}(t)=2^{-r} \rho_{r} f\left(\frac{t-t_{r}}{\rho_{r}} ; \beta\left(t_{r}\right)\right) .
$$

We prove first that

$$
\beta\left(t_{0} ; F\right) \leq \beta\left(t_{0}\right),
$$

for each point $t_{0}$. For every $h>0$, the interval

$$
t_{0}-h<t<t_{0}+h
$$

has a subinterval $I_{r}$ which contains the point $t_{0}$. Since

$$
\beta\left(t_{r}\right) \leq \beta\left(t_{0}\right),
$$


and since the function $F(t)$ does not satisfy in $I_{r}$ a Lipschitz condition with an exponent greater than $\beta\left(t_{r}\right)$, the relation $(15)$ is established.

Secondly, we show that

$$
\beta\left(t_{0} ; F\right) \geq \beta\left(t_{0}\right)-\epsilon
$$

for each point $t_{0}$ and every $\epsilon>0$. Because $\beta(t)$ is lower semi-continuous,

$$
\beta(t)>\beta\left(t_{0}\right)-\epsilon
$$

in some interval $\left|t-t_{0}\right|<\delta$. We choose a pair of integers $r_{1}$ and $r_{2}\left(r_{2}>r_{1}\right)$ such that

$$
t_{0}-\delta<t_{r_{1}}<t_{0}<t_{r_{2}}<t_{0}+\delta
$$

and denote by $l$ the closed interval $\left(t_{r_{1}}, t_{r_{2}}\right)$. We make separate estimates for those terms $f_{r}(t)$ for which $t_{r}$ lies in $l$, for those terms with $r>r_{2}$ for which $t_{r}$ is exterior to $I$, and for those terms with $r<r_{2}$ for which $t_{r}$ is exterior to $I$.

By (13) and (14), the inequality

$$
\left|f_{r}\left(t^{\prime \prime}\right)-f_{r}\left(t^{\prime}\right)\right| \leq 2^{1-r}\left|t^{\prime \prime}-t^{\prime}\right|^{\beta\left(t_{r}\right)}
$$

holds for all distinct $t^{\prime}$ and $t^{\prime \prime}$. This inequality implies that the sum of all terms $f_{r}(t)$ for which $t_{r}$ lies in $I$ satisfies throughout $I$ a Lipschitz condition with exponent $\beta\left(t_{0}\right)-\epsilon$. If $r>r_{2}$ and if $t_{r}$ does not lie in $I$, then $f_{r}^{\prime}(t)<2^{r-1}$ in $I$, so that the sum of all terms $f_{r}(t)$ corresponding to such values of $r$ has a bounded derivative in $l$, that is, satisfies throughout $I$ a Lipschitz condition with exponent 1. Finally, let $l^{\prime}$ be a subinterval of $l$ containing $t_{0}$ and sufficiently small to exclude all points $t_{r}$ for which $r<r_{2}$, except those coinciding with $t_{0}$. The sum of the corresponding terms $f_{r}(t)$ also has a bounded derivative in $I^{\prime}$. The inequality $(16)$ is established, and the proof of Theorem 5 is complete.

\section{REFERENCES}

1. J. H. Curtis et al., Amer. Math. Monthly 47 (1940), p.53, and Amer. Math. Monthly 48 (1941), pp. $413-414$.

2. W. S. Loud, Functions with prescribed Lipschitz condition, Proc. Amer. Math. Soc. 2 (1951), pp. $358-360$. 



\section{PACIFIC JOURNAL OF MATHEMATICS}

\section{EDITORS}

\section{R. U. FOEINSON}

University of California

Berkeley 4, California

E. HewitT

University of Washington

Seattle 5 , Washington
P. P. DILWOR TH

California Institute of Technology

Pasadena 4, California

E. F. BECKENBACH

University of California

Los Angeles 24, California

\section{ASSOCIATE EDITORS}

$\begin{array}{llll}\text { H. BUSEMANN } & \text { P. R. HALMOS } & \text { BØRGE JESSEN } & \text { J. J. STOKER } \\ \text { HERBERT FFDERER } & \text { IIEINZ HOPF } & \text { PAUL LÉVY } & \text { E. G. STRAUS } \\ \text { MARSHALL, IIALI } & \text { R. D. JAMES } & \text { GEORGE PÓLYA } & \text { KÖSAKU YOSIDA }\end{array}$

\section{SPONSORS}

UNIVERSITY OF BRITISH COLUMBIA

CALIFORNIA INSTITUTE OF TECHNOLOGY.

UNIVERSITY OF CAIJIFORNIA, BERKELEY

UNIVERSITY OF CAIJIFORNIA, DAVIS

UNIVERSITY OF CALIFORNIA, LOS ANGELES

UNIVERSITY OF CALIFORNI A, SANTA BARBARA

UNIVERSITY OF NEVADA

OREGON STATE COLLEGE

UNIVERSITY OF OREGON

\author{
UNIVERSITY OF SOU TIERN CALIFORNIA \\ STANFORD RESEARCH INSTITUTE \\ STANFORD UNIVERSITY \\ WASHINGTON STATE COLLEGE \\ UNIVERSITY OF WASHINGTON \\ AMERICAN MATHEMATICAL SOCIETY \\ NATIONAL BUREAU OF STANDARDS, \\ INSTITUTE FOR NUMERICAL ANALYSIS
}

Mathematical papers intended for publication in the Pacific Journal of Mathematics should be typewritten (double spaced), and the author should keep a complete copy. Manuscripts may be sent to any of the editors except Robinson, whose term expires with the completion of the present volume; they might also be sent to M.M. Schiffer, Stanford University, Stanford, California, who is succeeding Robinson. All other communications to the editors should be addressed to the managing editor, E. F. Beckenbach, at the address given above.

Authors are entitled to receive 100 free reprints of their published papers and may obtain additional copies at cost.

The Pacific Journal of Mathematics is published quarterly, in March, June, September, and December. The price per volume (4 numbers) is $\$ 8.00$; single issues, $\$ 2.50$. Special price to individual faculty members of supporting institutions and to individual members of the American Mathematical Society: $\$ 4.00$ per volume; single issues, $\$ 1.25$.

Subscriptions, orders for back numbers, and changes of address should be sent to the publishers, University of California Press, Berkeley 4, California.

Printed at Ann Arbor, Michigan. Entered as second class matter at the Post Office, Berkeley, California.

\section{UNIVERSITY OF CALIFORNIA PRESS • BERKELEY AND LOS ANGELES}




\section{Pacific Journal of Mathematics}

\section{Vol. 3, No. 2 \\ April, 1953}

William George Bade, An operational calculus for operators with spectrum

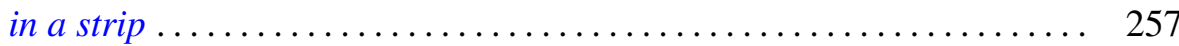

E. F. Beckenbach and Lloyd Kenneth Jackson, Subfunctions of several variables ..................................... 291

David Blackwell, Extension of a renewal theorem ................. 315

L. Carlitz, Some theorems on the Schur derivative ................ 321

Paul Arnold Clement, Generalized convexity and surfaces of negative curvature..................................... 333

Merrill M. Flood, On the Hitchcock distribution problem ............... 369

Watson Bryan Fulks, On the unique determination of solutions of the heat

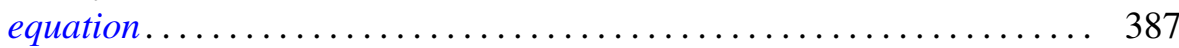

John W. Green, Length and area of a convex curve under affine transformation .................................... 393

William Gustin, An isoperimetric minimax .................. 403

Arthur Eugene Livingston, Some Hausdorff means which exhibit the Gibbs'

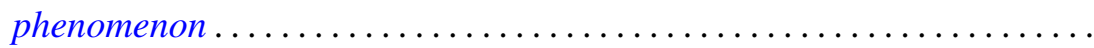

Charles Loewner, On generation of solutions of the biharmonic equation in the plane by conformal mappings ..................... 417

Gábor Szegő, Remark on the preceding paper of Charles Loewner ....... 437

Imanuel Marx and G. Piranian, Lipschitz functions of continuous

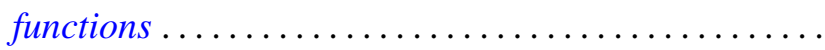

Ting-Kwan Pan, The spherical curvature of a hypersurface in Euclidean space ..

Ruth Lind Potter, On self-adjoint differential equations of second order ...

E. H. Rothe, A note on the Banach spaces of Calkin and Morrey...

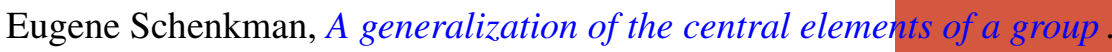

A. Seidenberg, A note on the dimension theory of rings .. . . 